\title{
Telavancin in the treatment of invasive Gram-positive infections
}

This article was published in the following Dove Press journal:

Clinical Audit

31 October 2012

Number of times this article has been viewed

\author{
Yoav Keynan ${ }^{1-4}$ \\ Ethan Rubinstein ${ }^{2,3}$ \\ 'Department of Community Health \\ Sciences, ${ }^{2}$ Department of Medical \\ Microbiology, ${ }^{3}$ Department of Internal \\ Medicine, University of Manitoba, \\ Winnipeg, Canada; ${ }^{4}$ Department \\ of Medical Microbiology, University \\ of Nairobi, Nairobi, Kenya
}

\begin{abstract}
Telavancin is a newer once-daily lipoglycopeptide with activity against Gram-positive bacteria, including those that are resistant to conventional antibiotics like beta-lactams. The results of recent clinical trials led to registration of telavancin for use in skin and skin structure infections in the US and Canada and for nosocomial pneumonia in Europe, based primarily on the favorable results of clinical trials. We review the evidence for use of telavancin in Gram-positive intravascular infections, focusing on bacteremic subpopulations in the large clinical trials as well as anecdotal evidence for use of the drug in the setting of infective endocarditis.
\end{abstract}

Keywords: telavancin, Gram-positive bacteria, skin infections, infective endocarditis

\section{Introduction}

Gram-positive bacteria are common causes of both community-acquired and nosocomial invasive infections, and are a leading cause of bloodstream infections. Staphylococcus aureus is a leading cause of bacteremia, and has become more common in recent years with the increase in invasive procedures, indwelling catheters, and prosthetic devices, as well as the aging population. These infections are associated with substantial morbidity, complications, and mortality. The increase in methicillinresistant $S$. aureus (MRSA) both in the health care setting and the recent explosive rise in community-acquired MRSA has further complicated the management of patients with staphylococcal bacteremia, increasing the duration of hospital stay and decreasing the availability of clinically effective therapeutic agents, and has been accompanied by increased costs of hospital stay and patient management. ${ }^{1-4}$

Telavancin is an intravenous lipoglycopeptide agent, with an improved tissue distribution profile supporting once-daily dosing without the need for serum concentration monitoring. ${ }^{5}$ Telavancin is a 14 times more potent inhibitor of peptidoglycan synthesis than vancomycin. In addition, telavancin has an additional mechanism of action that sets it apart from other glycopeptide derivatives, namely, it interacts selectively with the bacterial cell membrane, leading to an increase in membrane permeability with ensuing efflux of intracellular ATP and potassium, and the ability to abolish the cell membrane potential. ${ }^{6,79}$ In vitro, telavancin is rapidly bactericidal against clinically important Gram-positive bacteria, ${ }^{11}, 12$ including MRSA, vancomycin-intermediate and vancomycin-resistant $S$. aureus, and penicillin-resistant Streptococcus pneumoniae. It is also active against beta-hemolytic streptococci, Listeria monocytogenes, Bacillus anthracis, and Corynebacterium spp..$^{8-13,16-19}$ In addition, telavancin maintains
Correspondence: Ethan Rubinstein Section of Infectious Diseases, Faculty of Medicine, 543-745 Bannatyne Avenue, 50 I Basic Sciences Building, Winnipeg, MB R3E 0J9, Canada

Tel + I 2049775680

Fax + I 2047893926

Emailerubins@yahoo.com 
antibacterial activity against various anaerobic Gram-positive organisms, such as lactobacilli, actinomycetes, clostridia, and Propionibacterium spp. ${ }^{8,13,16}$ In addition, telavancin retains bactericidal activity against vancomycin-tolerant ${ }^{13}$ and vancomycin-intermediate ${ }^{10,12,14,16,18}$ strains. Moreover, telavancin has been shown to possess a 2-4-fold greater antistaphylococcal potency compared with vancomycin, a 4-8-fold greater potency than linezolid, and is 2-fold more potent than daptomycin against $S$. aureus. ${ }^{13,14,19}$ Its potency is similar to oxacillin against methicillin-susceptible strains.

Telavancin is approved in the US and Canada for the treatment of adult patients with complicated skin and skin structure infections due to susceptible-Gram-positive pathogens. ${ }^{20}$ It is being evaluated in the US currently for registration in the management of Gram-positive nosocomial pneumonia. In Europe, telavancin has been approved for use in nosocomial pneumonia (including ventilator-associated pneumonia) caused by $S$. aureus. The role of $S$. aureus in hospital-acquired pneumonia, and specifically the fact that over $60 \%$ of isolates from US hospitals are resistant to methicillin and therapy is initiated prior to microbiological diagnosis, necessitates the use of antimicrobials with anti-MRSA activity prior to microbiological identification of the causative organism implicated in the pneumonia. ${ }^{21-26}$ Vancomycin and linezolid are recommended in current guidelines but have limitations, ${ }^{25,26}$ while tigecycline is not indicated in ventilator-associated pneumonia and daptomycin is not indicated for hospital-acquired pneumonia. ${ }^{22,26}$ Recently, the results of the ATTAIN (Assessment of Telavancin for Treatment of Hospital-Acquired Pneumonia) studies were reported..$^{25,28}$ These studies were international, randomized, double-blind, parallel-group Phase III trials investigating the efficacy and safety of telavancin in the treatment of hospital-acquired pneumonia caused by Gram-positive pathogens in adults. $S$. aureus was the most commonly isolated organism; two thirds of such isolates in the study were MRSA, reflecting the widespread occurrence of this organism globally. Comparison of telavancin with vancomycin yielded similar cure rates for pooled analysis. Telavancin was superior to vancomycin in patients with monomicrobial pneumonia due to $S$. aureus and in the subgroup of patients infected with $S$. aureus and a vancomycin minimum inhibitory concentration $\geq 1 \mu \mathrm{g} / \mathrm{mL}$ and trended towards superiority for monomicrobial MRSA pneumonia. The evidence for the role of telavancin in the treatment of Gram-positive bloodstream and intravascular infections is the focus of this review.

\section{Telavancin for treatment of bacteremia}

Telavancin has theoretical advantages compared with vancomycin. It is more rapidly bactericidal and has more potent activity against Gram-positive organisms, including MRSA and glycopeptide-intermediate S. aureus D, E-1. Because vancomycin is only slowly bactericidal against methicillin-sensitive $S$. aureus and linezolid is only bacteriostatic, telavancin and daptomycin remain the only options for reliable treatment of methicillin-sensitive $S$. aureus bacteremia in individuals who are allergic to beta-lactam antibiotics and those with MRSA bacteremia. These salutary effects of telavancin may be attributed to the dual action of the drug. In mouse models of heteroresistant vancomycin-intermediate and glycopeptide-intermediate $S$. aureus infection, telavancin has been shown to possess an 8-16-fold greater potency than vancomycin for different resistant strains. ${ }^{15,17,27,30,31,38}$ In a model using immunocompromised mice inoculated intraperitoneally with MRSA and treated with two subcutaneous doses (once every 12 hours) of vehicle or test compound, equivalent exposure to vancomycin and telavancin was ensured based on murine pharmacokinetic data. Telavancin produced significantly greater reductions in bacterial load compared with vancomycin in the blood and spleen. This reduction translated into significantly decreased mortality, from $100 \%$ in animals treated with vehicle and vancomycin to $7 \%$ in telavancin-treated animals. ${ }^{29}$

The ATLAS (Assessment of Telavancin in Complicated Skin and Skin Structure Infections) trials randomized 1867 patients with skin and skin structure infections to receive either vancomycin or telavancin. In the clinically evaluable population, microbiological and clinical success rates were similar for the two drugs at 7-14 days after the last antibiotic dose. A single causative pathogen was isolated in nearly $80 \%$ of participants and, of the isolated bacteria, $S$. aureus was the most common, accounting for $83 \%$ of monomicrobial infections. Although 56 patients in the ATLAS study had bacteremia (36 in the telavancin-treated group and 20 in the vancomycin-treated group) and $S$. aureus accounted for 34 cases, no separate analysis was reported for these bacteremic cases..$^{20,32}$

In a post hoc analysis of the ATTAIN studies, bacteremic hospital-acquired pneumonia was diagnosed based on the presence of a negative respiratory culture and recovery of a respiratory pathogen on baseline blood cultures, or in the 
setting where the same micro-organism, sharing the same antimicrobial susceptibility profile, was identified from both respiratory and blood samples at baseline. Seventy-three cases of bacteremia ( $6.7 \%$ of cases) were identified, comprising 34 in the telavancin group and 39 in the vancomycin group. Gram-positive pathogens were isolated from nearly $80 \%$ of patients and Gram-negative pathogens from roughly $25 \%$. S. aureus was the most frequent isolate, recovered from $72.6 \%$ of patients, with nearly two thirds being MRSA. The overall outcome at the test of cure assessment and pathogen eradication rates was similar. In the 33 patients with monomicrobial S. aureus infection, cure rates for telavancin and vancomycin patients were $60 \%$ and $33 \%$, respectively (Stryjewski, unpublished data, 2012).

\section{Telavancin for treatment of endocarditis}

$S$. aureus is the most common micro-organism associated with infective endocarditis, and due to an increase in the prevalence of MRSA, vancomycin is frequently used, despite its high rates of persistent bacteremia and failure. ${ }^{33,34}$ Evidence from in vitro and a rabbit model of aortic valve endocarditis comparing the activity of telavancin and vancomycin against methicillin-resistant and vancomycin-intermediate $S$. aureus strains provided some encouraging results. Telavancin was bactericidal in time-kill studies at a concentration of $5 \mu \mathrm{g} / \mathrm{mL}$ against both strains. Vancomycin was only bacteriostatic at the same concentration and bactericidal at $10 \mu \mathrm{g} / \mathrm{mL}$ against an MRSA strain, and was bacteriostatic at $10 \mu \mathrm{g} / \mathrm{mL}$ against the vancomycin-intermediate $S$. aureus strain. Compared with untreated control rabbits, a twice-daily regimen of telavancin $30 \mathrm{mg} / \mathrm{kg}$ reduced mean aortic valve vegetation titers of the MRSA strain after 4 days of therapy and sterilized six of 11 vegetations, compared with a lower reduction of colony-forming units and clearance of organisms from three of 10 vegetations on a regimen of twice-daily vancomycin $30 \mathrm{mg} / \mathrm{kg}$. Telavancin was significantly more effective than vancomycin in the vancomycin-intermediate $S$. aureus model, producing greater reduction versus no reduction in number of colony-forming units with vancomycin. ${ }^{18}$ Added to the potential for superior potency of telavancin in the setting of infection caused by Gram-positive pathogens, several lines of evidence point to an advantage in the context of biofilmproducing organisms. Telavancin has been shown to be more effective than vancomycin against biofilm-forming $S$. aureus in a pharmacokinetic filter model ${ }^{35}$ as well as in an in vitro biofilm activity assay. ${ }^{36}$ La Plante et al studied biofilm- producing reference strains of S. aureus, Staphylococcus epidermidis, and Enterococcus faecalis as well as biofilmproducing clinical isolates obtained from proven catheterrelated bloodstream infections. Vancomycin demonstrated no activity against bacteria embedded in a biofilm produced by $S$. aureus in contrast with telavancin that was shown to be active against these bacteria with a low minimum eradication concentration of 0.125 to $2 \mu \mathrm{g} / \mathrm{mL}$ for biofilm-embedded organisms, a concentration that is attainable with doses used clinically. They demonstrated the ability of telavancin to inhibit biofilm formation at concentrations below the respective minimum inhibitory concentration of each isolate. In contrast, vancomycin inhibited biofilm formation at concentrations only at or above the respective minimum inhibitory concentration of each isolate. ${ }^{36}$ Similarly, an in vivo rabbit model demonstrated that therapeutic doses of telavancin were superior to vancomycin for prevention of subcutaneous implant colonization and infection by $S$. aureus. The effect was concentration-dependent. ${ }^{37}$

In another animal model, telavancin was able to achieve a larger reduction in bacterial vegetation load, but the difference between vancomycin and telavancin failed to reach statistical significance. ${ }^{38}$ The first report of use of telavancin in rightsided MRSA endocarditis (vancomycin minimum inhibitory concentration $\leq 0.5 \mathrm{mg} / \mathrm{L}$, daptomycin minimum inhibitory concentration $\leq 1 \mathrm{mg} / \mathrm{L}$ ) described a patient in whom, despite a vancomycin dose of $15 \mathrm{mg} / \mathrm{kg}$ every 12 hours and trough levels of 15-20 mg/dL, blood cultures remained positive through 8 days of treatment. Treatment was then switched to telavancin intravenously every 24 hours with abrupt clearance of the bacteremia and a favorable outcome. ${ }^{39}$ A subsequent case report of vancomycin-intermediate $S$. aureus pacemaker lead endocarditis, that was initially daptomycin susceptible became resistant while on daptomycin (minimum inhibitory concentration initially $0.5 \mu \mathrm{g} / \mathrm{mL}$, increasing to $2.0 \mu \mathrm{g} /$ $\mathrm{mL})$. Treatment with telavancin resulted in rapid clearance of bacteremia within one day of initiation of telavancin and a favorable outcome. ${ }^{40} \mathrm{~A}$ similar case of telavancin use following unsuccessful treatment of $S$. aureus endocarditis has been reported. ${ }^{41}$ A case of failure of daptomycin to cure $S$. aureus endocarditis of the mitral valve was cured with subsequent telavancin and linezolid therapy, ${ }^{41}$ attesting to the utility of telavancin in severe infections.

\section{Summary}

Telavancin is a new addition to the treatment armamentarium for Gram-positive bacteria, with documented efficacy 
in skin and skin structure infections as well as in the setting of nosocomial pneumonia. S. aureus is a common cause of skin and skin structure infections and pneumonia, and is a leading cause of bacteremia and endocarditis. The rise in MRSA and its dissemination in the community, along with slow bactericidal activity of vancomycin, highlight the need for additional antimicrobial agents. Telavancin is one such antimicrobial agent, possessing theoretical benefits over vancomycin, both in terms of pharmacokinetics and in vitro data, particularly in the presence of a creeping vancomycin minimum inhibitory concentration. In addition, it has the potential for superior activity when biofilm formation is involved, such as that occurring in staphylococcal endocarditis. Analysis of the subsets of patients with bacteremia from the major trials leading to licensing of telavancin suggests that this drug is as effective as vancomycin, with potential superiority in the monomicrobial infections included in the ATTAIN trial. Experience with the use of telavancin for treatment of staphylococcal endocarditis is limited to a few case reports, but is promising given the demonstrated effect in biofilm models. Use of telavancin for the treatment of enterococcal infections remains to be determined, given that this organism is not one of the common culprits causing skin or pulmonary infections.

\section{Disclosure}

The authors report no conflicts of interest in this work.

\section{References}

1. Rice LB. Antimicrobial resistance in Gram-positive bacteria. Am J Med. 2006;119(6A):S11-S19.

2. Schmidt-Ioanas M, Roux A, Lode H. New antibiotics for the treatment of severe staphylococcal infection in the critically ill patient. Curr Opin Crit Care. 2005;11:481-486.

3. Lawes T, Edwards B, López-Lozano JM, Gould I. Trends in Staphylococcus aureus bacteremia and impacts of infection control practices including universal MRSA admission screening in a hospital in Scotland, 2006-2010: retrospective cohort study and time-series intervention analysis. BMJ Open. 2012;2:3.

4. Wolkewitz M, Frank U, Philips G, Schumacher M, Davey P; BURDEN Study Group. Mortality associated with in-hospital bacteraemia caused by Staphylococcus aureus: a multistate analysis with follow-up beyond hospital discharge. J Antimicrob Chemother. 2011;66:381-386.

5. Van Bambeke F. Glycopeptides in clinical development: pharmacological profile and clinical perspectives. Curr Opin Pharmacol. 2004;4: 471-478.

6. Higgins DL, Chang R, Debabov DV, et al. Telavancin, a multifunctional lipoglycopeptide, disrupts both cell wall synthesis and cell membrane integrity in methicillin-resistant Staphylococcus aureus. Antimicrob Agents Chemother. 2005;49:1127-1134.

7. Lunde CS, Hartouni SR, Janc JW, Mammen M, Humphrey PP, Benton BM. Telavancin disrupts the functional integrity of the bacterial membrane through targeted interaction with the cell wall precursor lipid II. Antimicrob Agents Chemother. 2009;53:3375-3383.
8. Goldstein EJ, Citron DM, Merriam CV, et al. In vitro activities of the new semisynthetic glycopeptide telavancin (TD-6424), vancomycin, daptomycin, linezolid, and four comparator agents against anaerobic Gram-positive species and Corynebacterium spp. Antimicrob Agents Chemother. 2004;48:2149-2152.

9. King A, Phillips I, Kaniga K. Comparative in vitro activity of telavancin (TD-6424), a rapidly bactericidal, concentration-dependent antiinfective with multiple mechanisms of action against Gram-positive bacteria. J Antimicrob Chemother. 2004;53:797-803.

10. Pace JL, Krause K, Johnston D, et al. In vitro activity of TD-6424 against Staphylococcus aureus. Antimicrob Agents Chemother. 2003;47: 3602-3604.

11. Draghi DC, Jones ME, Flamm RK, Thornsberry C, Sahm DF. Telavancin activity against current and diverse populations of enterococci and Streptococcus pneumoniae. Presented at the 45th Annual Interscience Conference on Antimicrobial Agents and Chemotherapy, December 16-19, 2005, Washington, DC.

12. Mendes RE, Sader HS, Farrell DJ, Jones RN. Worldwide appraisal and update (2010) of telavancin activity tested against a collection of Gram-positive clinical pathogens from five continents. Antimicrob Agents Chemother. 2012;56:3999-4004.

13. Song Y, Lunde CS, Benton BM, Wilkinson BJ. Further insights into the mode of action of the lipoglycopeptide telavancin through global gene expression studies. Antimicrob Agents Chemother. 2012;56: 3157-3164.

14. Leuthner KD, Cheung CM, Rybak MJ. Comparative activity of the new lipoglycopeptide telavancin in the presence and absence of serum against 50 glycopeptide non-susceptible staphylococci and three vancomycin-resistant Staphylococcus aureus. JAntimicrob Chemother. 2006;58:338-343.

15. Finegold SM, Bolanos M, Summanen P, Molitoris DR. In vitro activity of telavancin and six comparator agents against 460 anaerobic bacteria. Presented at the 45th Annual Interscience Conference on Antimicrobial Agents and Chemotherapy, December 16-19, 2005, Washington, DC.

16. Eliopoulos GM, Wennersten CB, Sakoulas G, Moellering RC Jr. In vitro bactericidal activity of telavancin against $S$. aureus with relative tolerance to vancomycin. Presented at the 44th Annual International Conference on Antimicrobial Agents and Chemotherapy, October 30November 2, 2004, Washington, DC.

17. Madrigal AG, Basuino L, Chambers HF. Efficacy of telavancin in a rabbit model of aortic valve endocarditis due to methicillin-resistant Staphylococcus aureus or vancomycin-intermediate Staphylococcus aureus. Antimicrob Agents Chemother. 2005;49:3163-3165.

18. Draghi DC, Benton BM, Krause KM, Thornsberry C, Pillar C, Sahm DF. Comparative surveillance study of telavancin activity against recently collected Gram-positive clinical isolates from across the United States. Antimicrob Agents Chemother. 2008;52:2383-2388.

19. Jansen WT, Verel A, Verhoef J, Milatovic D. In vitro activity of telavancin against Gram-positive clinical isolates recently obtained in Europe. Antimicrob Agents Chemother. 2007;51:3420-3424.

20. Stryjewski ME, Graham DR, Wilson SE. Telavancin versus vancomycin for the treatment of complicated skin and skin-structure infections caused by Gram-positive organisms. Clin Infect Dis. 2008;46:1683-1693.

21. American Thoracic Society/Infectious Diseases Society of America: Guidelines for the management of adults with hospital-acquired, ventilator associated and healthcare associated pneumonia. Am J Respir Crit Care. 2005;171:388-416.

22. Rotstein C, Evans G, Born A, et al. Clinical practice guidelines for hospital-acquired pneumonia and ventilator-associated pneumonia in adults. Can J Infect Dis Med Microbiol. 2008;19:19-53.

23. Torres A, Ewig S, Lode H, Carlet J. Hospital-acquired pneumonia in Europe. Eur Respir J. 2009;33:951-952.

24. Ramirez P, Fernández-Barat L, Torres A. New therapy options for MRSA with respiratory infection/pneumonia. Curr Opin Infect Dis. 2012;25:159-165. 
25. Rubinstein E, Lalani T, Corey GR. Telavancin versus vancomycin for hospital-acquired pneumonia due to Gram-positive pathogens. Clin Infect Dis. 2011;52:31-40.

26. Hooper CY, Smith WJ. Telavancin for the treatment of nosocomial pneumonia caused by methicillin-resistant Staphylococcus aureus (MRSA). Ther Clin Risk Manag. 2012;8:131-137.

27. Marconescu P, Graviss EA, Musher DM. Rates of killing of methicillinresistant Staphylococcus aureus by ceftaroline, daptomycin, and telavancin compared to that of vancomycin. Scand J Infect Dis. 2012;44: $620-622$.

28. Rubinstein E, Corey GR, Stryjewski ME, Kanafani ZA. Telavancin for the treatment of serious Gram-positive infections, including hospital acquired pneumonia. Expert Opin Pharmacother. 2011;12: 2737-2750.

29. Hegde SS, Difuntorum S, Skinner R, Trumbull J, Krause KM. Efficacy of telavancin against glycopeptide-intermediate Staphylococcus aureus in the neutropenic mouse bacteraemia model. JAntimicrob Chemother. 2009;63:763-766.

30. Hegde SS, Skinner R, Lewis SR, Krause KM, Blais J, Benton BM. Activity of telavancin against heterogeneous vancomycin-intermediate Staphylococcus aureus (hVISA) in vitro and in an in vivo mouse model of bacteraemia. J Antimicrob Chemother. 2010;65:725-728.

31. Reyes N, Skinner R, Benton BM, et al. Efficacy of telavancin in a murine model of bacteraemia induced by methicillin-resistant Staphylococcus aureus. J Antimicrob Chemother. 2006;58:462-465.

32. Stryjewski ME, Barriere SL, O'Riordan W, et al. Efficacy of telavancin in patients with specific types of complicated skin and skin_structure infections. J Antimicrob Chemother. 2012;67:1496-1502.

33. Fowler VG, Miro JM, Hoen B, et al. Staphylococcus aureus endocarditis: a consequence of medical progress. JAMA. 2005;293:3012-3021.
34. Miro JM, Anguera I, Cabell CH, et al; International Collaboration on Endocarditis Merged Database Study Group. Staphylococcus aureus native valve infective endocarditis: report of 566 episodes from the International Collaboration on Endocarditis Merged Database. Clin Infect Dis. 2005;41:507-514.

35. Gander S, Kinnaird A, Finch R. Telavancin: in vitro activity against staphylococci in a biofilm model. J Antimicrob Chemother. 2005;56: 337-343.

36. LaPlante KL, Mermel LA. In vitro activity of daptomycin and vancomycin lock solutions on staphylococcal biofilms in a central venous catheter model. Nephrol Dial Transplant. 2007;22:2239-2246.

37. Darouiche RO, Mansouri MD, Schneidkraut MJ. Comparative efficacies of telavancin and vancomycin in preventing device-associated colonization and infection by Staphylococcus aureus in rabbits. Antimicrob Agents Chemother. 2009;53:2626-2628.

38. Miro JM, Garcia-de-la-Maria C, Armero Y, et al. Efficacy of telavancin in the treatment of experimental endocarditis due to glycopeptideintermediate Staphylococcus aureus. Antimicrob Agents Chemother. 2007;51:2373-2377.

39. Nace H, Lorber B. Successful treatment of methicillin-resistant Staphylococcus aureus endocarditis with telavancin. J Antimicrob Chemother. 2010;65:1315-1316.

40. Marcos LA, Camins BC. Successful treatment of vancomycin-intermediate Staphylococcus aureus pacemaker lead infective endocarditis with telavancin. Antimicrob Agents Chemother. 2010;54:5376-5378.

41. Joson J, Grover C, Downer C, Pujar T, Heidari A. Successful treatment of methicillin-resistant Staphylococcus aureus mitral valve endocarditis with sequential linezolid and telavancin monotherapy following daptomycin failure. J Antimicrob Chemother. 2011;66:2186-2188.
Clinical Audit

\section{Publish your work in this journal}

Clinical Audit is an international, peer-reviewed, open access journal focusing on the processes and outcomes of clinical audit in any area of healthcare. All aspects of patient care are addressed within the journal and practitioners from all disciplines are invited to submit their work. Areas covered include: Publication of audits; How an audit has changed practice;

\section{Dovepress}

Practical tips on how to do audits and to avoid pitfalls; How audits have changed patient care; Calls and justifications for new audits. The manuscript management system is completely online and includes a very quick and fair peer-review system, which is all easy to use. Visit http://www.dovepress. com/testimonials.php to read real quotes from published authors. 